\title{
A novel tool for biochemical diagnostics of rare genetic disorders: an integrated microfluidic system with optical detection
}

\author{
Radoslaw Kwapiszewski, Karina Ziolkowska, Zbigniew Brzozka \\ Department of Microbioanalytics, Institute of Biotechnology, Faculty of Chemistry, Warsaw University \\ of Technology, Noakowskiego 3, 00-664 Warsaw, Poland \\ brzozka@ch.pw.edu.pl
}

\begin{abstract}
In this work, an integrated microfluidic system for biochemical diagnostics of lysosomal storage disorders is presented. The polymeric microsystem consists of a zone for hydrodynamic focusing of cell suspension, a mixing microchannel, and an optical detection module. The system was used for determination of the activity of enzymes deficient in Fabry and Gaucher diseases. On the contrary to the currently used protocols of determination of lysosomal enzymes' activities, the microdevice enables significant reduction of the time of analysis. Moreover, the experimental set enables to avoid termination of enzymatic reaction and sample dilution, what increases sensitivity of the developed method. Due to easy fabrication steps and their low cost, the system seems to be a prospective tool for a point-of-care approach.
\end{abstract}

Key words: microfluidics, lab-on-a-chip, optical detection, laboratory diagnostics, lysosomal storage disorders

\section{Introduction}

Microfluidics and biosensors for life sciences and chemistry are current fields of interests of many scientists worldwide. These approaches have emerged for improving performance and functionality of biochemical and medical analysis [1]. Apart from low reagents consumption, short reaction time, versatility, possibility of automation, continuous monitoring and stimulation of each step of the procedure, the use of miniaturized microdevices in progressive clinical medicine (point of care systems, POCS) affects the patients' comfort [2].

Currently, more than 3000 congenital metabolic diseases are known, e.g. mitochondrial, peroxisomal, lysosomal. Lysosomal storage disorders (LSDs) are a group of more than 45 rare inherited metabolic diseases, i.e. Fabry, Gaucher, Niemann-Pick, Pompe or Tay-Sachs diseases, that result from defects in lysosomal function [3]. Most of them are caused by abnormal activity of a proper enzyme. Currently applied methods, which are based on determination of enzyme activity, are timeconsuming, inaccurate (there are many falsepositive and false-negative test results), and are carried out only in a few laboratories in the world [4].

The goal of this work was to design and develop a microfluidic system for laboratory diagnostics of lysosomal storage disorders. The usability of the microsystem was verified by performing assays of $\alpha$-galactosidase activities (GLA, deficient in Fabry disease) and $\beta$-glucocerebrosidase (GBA, deficient in Gaucher disease) using Jurkat T-cells as a source of enzymes.

\section{Experimental}

The microsystem was fabricated in poly(dimethylsiloxane) (PDMS) by the replica molding technique using a micromilled poly(methyl methacrylate) (PMMA) master. The microsystem consists of a zone for hydrodynamic focusing of cell suspension, a mixing microchannel, and an optical detection module connected with an external light source and a detector by optical fibers (see Fig. 1).

The $150 \mu \mathrm{m}$ wide and $300 \mu \mathrm{m}$ high micromixing channel is formed by three $50 \mu \mathrm{m}$ wide and $300 \mu \mathrm{m}$ high microchannels. Due to a large contact area between the flowing streams, the mixing of the reagents is efficient. Application of a smooth micromixing channel prevents from accumulation of compounds and cells' 
membranes, what is observed in case of using a slanted or herringbone microchannel [5].

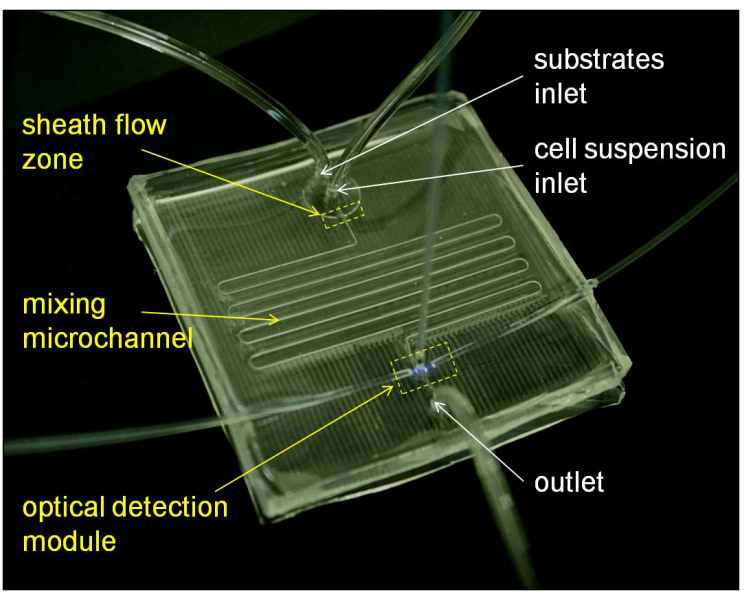

Fig. 1. A view of the integrated microsystem.

Fabrication of the detection module based on a special alignment of optical fibers requires using of a holder (see Fig. 2). The holder for precise positioning of optical fibers using grooves was manufactured in PMMA. Two exciting quartz fibers $(600 / 950 \mu \mathrm{m})$ were embedded in PDMS during molding at $20-25^{\circ}$ angle to the surface, and one collecting quartz fiber $(600 / 950 \mu \mathrm{m})$ was placed orthogonally to the widened microchannel. Such optical fibers' setting enabled to avoid high background fluorescence, greatly reducing the chance of reaching the detector by excitation light, and in consequence, enabled detection of the fluorescent product even within the first minutes of the enzymatic reactions.

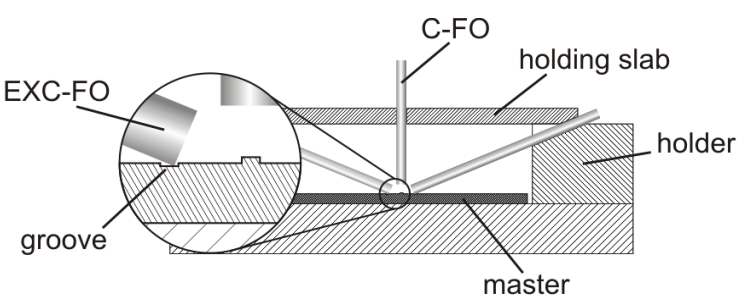

Fig. 2. The holder for precise positioning of the exciting optical fibers (EXC-FO) and the collecting optical fiber (C-FO).

\section{Results and discussion}

A principle of the determination of $\alpha$-galactosidase and $\beta$-glucocerebrosidase activities was fluorometric measurement of a protonated form of the product (4-methylumbelliferone) released in the enzymatic reactions (see Fig. 3). The concentration of the released product was determined against a 4-methylumbelliferone standard curve. The curve of advancement of the enzymatic reaction performed in the presented microsystem (see Fig. 4) was estimated for different reaction times obtained by the change of the cell suspension and substrates flow rates maintained in the constant 1:4 ratio (see Table 1 ). The ratio was optimized to obtain a width of a focused stream with cell suspension on the order of $15 \mu \mathrm{m}$ (matched to a diameter of mammalian cells).

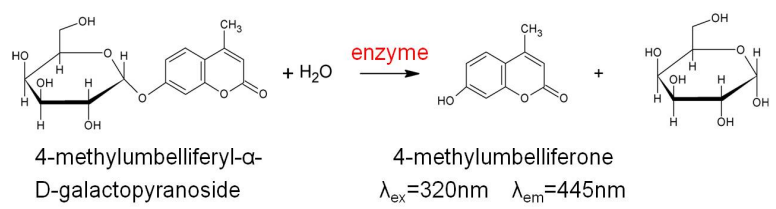

Fig. 3. The reaction catalyzed by $\alpha$-galactosidase.

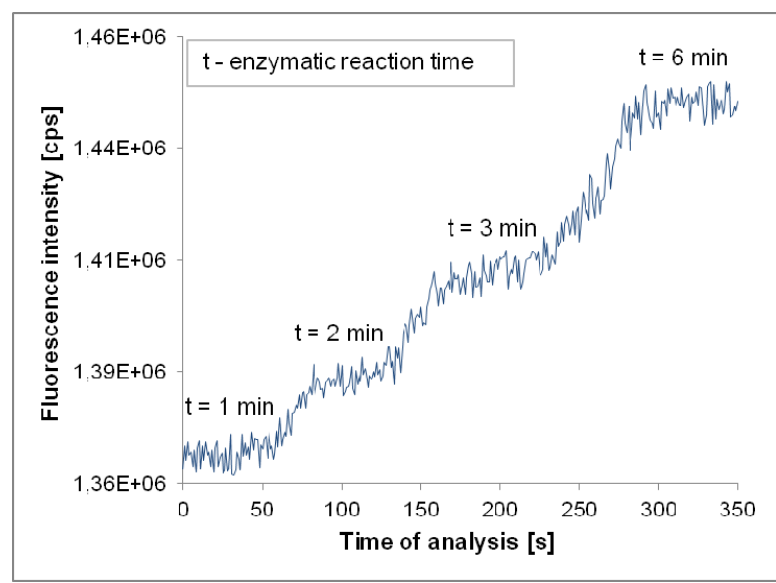

Fig. 4. The curve of advancement of the enzymatic reaction carried out in the microsystem.

Determinations of $\alpha$-galactosidase and $\beta$-glucocerebrosidase activities in the presented microsystem were performed using Jurkat Tcells suspension as leucocytes are commonly used for biochemical diagnostics of Fabry and Gaucher diseases. The calculated GLA and GBA activities were $4.18 \pm 0.52 \mu \mathrm{U} / 10^{6}$ cells and $2.19 \pm 0.31 \mu \mathrm{U} / 10^{6}$ cells, respectively. Relatively low values of the determined standard deviations in both cases $(S D<15 \%)$ indicate high repeatability of the measurements. Detection of the fluorescent product released even after the first minute of the enzymatic reaction was possible because of the special arrangement of the optical fibers and a flow analysis. The flow analysis enabled to avoid the termination of the enzymatic reaction with alkaline buffer (termination of an enzymatic reaction leads to dilution of a sample decreasing sensitivity of a method). In consequence, the total time of analysis using the presented microsystem was $10 \mathrm{~min}$, whereas the macro-scale protocols of the GLA and GBA activities determination are highly time-consuming (only the sample incubation step lasts even 2 hours [6]). Due to the observed effect of a surface-to-volume ratio on fluorescence-based assays [7], it was hard to compare obtained results with macro-scale results following the standard protocols. 
Tab. 1

\begin{tabular}{|c|c|c|}
\hline $\begin{array}{c}\text { Cell } \\
\text { suspension } \\
\text { flow rate } \\
{[\mu \mathrm{L} / \mathrm{min}]}\end{array}$ & $\begin{array}{c}\text { Substrates } \\
\text { flow rate } \\
{[\mu \mathrm{L} / \mathrm{min}]}\end{array}$ & $\begin{array}{c}\text { Enzymatic } \\
\text { reaction time } \\
{[\mathrm{min}]}\end{array}$ \\
\hline 3 & 12 & 1 \\
\hline 1.5 & 6 & 2 \\
\hline 1 & 4 & 3 \\
\hline 0.5 & 2 & 6 \\
\hline
\end{tabular}

\section{Conclusion}

In this work, the possibility for determination of intracellular enzymes' activities using the microfluidic system with optical detection is presented. The activities of $\alpha$-galactosidase and $\beta$-glucocerebrosidase deficient in Fabry and Gaucher diseases diagnostics, respectively, using Jurkat T-cells were determined. Due to a good sensitivity of the method and short time of analysis $(10 \mathrm{~min})$, the presented microsystem, which is relatively cheap and easy to fabrication, seems to be a prospective tool for a point-of-care approach.

Future works should address integration of a source of light into a microsystem to make it a portable tool. Works should be also focused on determination of GLA, GBA and other lysosomal enzymes activities using real samples. After the validation of the analytical procedure the presented microsystem will be ready for preliminary tests in clinical laboratories.

\section{Acknowledgements}

The project is realized within the MPD programme of Foundation for Polish Science, co-financed from European Union, Regional Development Fund Grants for innovation.

\section{References}

[1] N.-T. Nguyen, S.T. Wereley, Fundamentals and applications of microfluidics. Artech House, Inc. Boston, London (2002)

[2] R. Kwapiszewski, M. Skolimowski, K. Ziółkowska, E. Jędrych, M. Chudy, A. Dybko, Z. Brzózka, Biomedical Microdevices 13, 431-440 (2011); doi: 10.1007/s10544-011-9511-0

[3] G.M. Pastores, Lysosomal Storage Disorders: Principles and Practice. World Scientific Publishing Company (2009)

[4] R.J. Desnick, R. Brady, J. Barranger, A.J. Collins D.P. Germain, M. Goldman, G. Grabowski, S. Packman, W.R. Wilcox, Annals of Internal Medicine 138, 338-346 (2003)
[5] A.D. Stroock, S.K.W. Dertinger, A. Ajdari, I. Mezic, H.A. Stone, G.M. Whitesides, Science 295, 647-651 (2002); doi: 10.1126/science. 1066238

[6] A.M. Martins, V. D'Almeida, S.O. Kyosen, E.T. Takata, A.G. Delgado, A.M. Gonçalves, C.C. Benetti Filho, D. Martini Filho, G. Biagini, H. Pimentel, H. Abensur, H.C. Guimarães, J.G. Gomes, J. Sobral Neto, L.O. D'Almeida, L.R. Carval-ho, M.B. Harouche, M.C. Maldonado, O.J. Nascimento, P.S. Montoril, R.V. Bastos, Journal of Pediatrics 155 (4 Suppl.), S19-S31 (2009); doi: 10.1016/j.jpeds.2009.07.003

[7] R. Kwapiszewski, K. Ziolkowska, K. Zukowski, M. Chudy, A. Dybko, Z. Brzozka, Analytical and Bioanalytical Chemistry 403, 151-155 (2012); doi: $10.1007 / \mathrm{s} 00216-012-5770-8$ 